\title{
REFLEXÕES DA ENFERMAGEM NO MANEJO AO PACIENTE IDOSO COM DELIRIUM EM TERAPIA INTENSIVA ${ }^{1}$
}

\author{
NURSING REFLECTIONS IN THE MANAGEMENT OF THE \\ ELDERLY PATIENT WITH DELIRIUM IN INTENSIVE THERAPY
}

\author{
Marcos José Ferreira², Angélica Garcia dos Passos ${ }^{3}$, \\ Wellington Fernando da Silva Ferreira ${ }^{4}$ e Elia Machado de Oliveira ${ }^{5}$
}

\section{RESUMO}

O distúrbio psiquiátrico comum em pacientes idosos hospitalizados é o delirium, que consiste em um estado de confusão agudo e retrata a manifestação da disfunção cerebral. Desta forma, os profissionais da enfermagem são fundamentais na identificação de fatores de risco, aplicando condutas baseadas em evidências, colaborando para a melhoria no quadro do paciente idoso na unidade de terapia intensiva. Trata-se de uma revisão integrativa de literatura de caráter exploratório, com abordagem qualitativa, na qual realizou-se a busca de estudos em periódicos nacionais em base de dados: SCIELO, LILACS, BIREME, publicados entre os anos de 2014 ao primeiro trimestre de 2018, com amostra final constituída por 21 artigos científicos completos. Os achados apontam trabalhos científicos voltados a eixos fundamentais, e demonstram intercessão entre aspectos farmacológicos e não farmacológicos, tendo o delirium, relação direta com agravamento de comorbidades. Contudo, o reconhecimento dos fatores de risco não modificáveis e de possível intervenção são estratégias fundamentais da prevenção do delirium em idosos a âmbito de unidade de terapia intensiva. A identificação dos sinais advinda dos idosos é de fundamental importância para sua rápida intervenção e diagnóstico, para que sejam propostas condutas aos episódios e estabelecidas ações de vigilância contínua. Portanto, com a reflexão desta temática, faz-se necessária a combinação das atribuições da enfermagem e a contribuição para a compreensão da incidência e prevalência da síndrome, proporcionando clarear a importância dos fatores de risco e estratégias de prevenção modificáveis do delirium em unidade de terapia intensiva.

Palavras-chave: Pessoa Idoso, Delirium, Unidades de terapia intensiva, Enfermagem.

\section{ABSTRACT}

The most common psychiatric disorder in hospitalized elderly patients is delirium, which consists of a state of acute confusion and depicts the manifestation of brain dysfunction. That way, nursing professionals are essential in the identification of risky factors using the prevention strategies based in evidences, collaborating for the improvement in the elderly patient status when in the ICU. This is a systematic exploratory literature review of a qualitative approach, in which it has researched for studies in national journals in databases: SCIELO, LILACS, BIREME published between the years of 2014 and the first half of 2018, with a final sample consisting

\footnotetext{
${ }^{1}$ Trabalho de Conclusão de Curso de Bacharel em Enfermagem.

${ }^{2}$ Graduado Bacharel em Enfermagem - UNIANDRADE - Centro Universitário Campos de Andrade. E-mail: marfer@ gmail.com

${ }^{3}$ Graduada Bacharel em Enfermagem - UNIANDRADE - Centro Universitário Campos de Andrade. E-mail: anja@gmail.com

${ }^{4}$ Colaborador - Enfermeiro, Especialista em Saúde do Idoso e Gerontologia, Mestrando em Saúde Coletiva pela Universidade Federal do Paraná - UFPR. E-mail: wellington.ferreira@ufpr.br

${ }^{5}$ Orientadora - Enfermeira, Mestre em Cirurgia - PUCPR, Docente da Universidade Estácio de Sá - Curitiba. E-mail: elia561@ hotmail.com
} 
of 21 complete scientific articles. The findings point out to scientific studies focused on fundamental axes, and demonstrate an intercession between pharmacological and non-pharmacological aspects, with delirium being a direct relation with aggravation of comorbidities. However, the recognition of the non modifiable and modifiable risk factors of possible intervention is an essential strategy for the prevention of delirium in the elderly in ICUs. The identification of the signs coming from the elderly, is of fundamental importance to its rapid intervention and diagnosis, so that there is the proposal of the conducts to the episodes, establishing actions of continuous surveillance. Therefore, with the reflection of this theme, it is necessary to link the nursing attributions for contributing to the understanding of the incidence and prevalence of the syndrome, providing clarification of the importance of modifiable risk factors and prevention strategies of delirium in ICU.

Keywords: Elderly Person, Delirium, Intensive Care Units, Nursing.

\section{INTRODUÇÃO}

O envelhecimento populacional vem crescendo mundialmente com o passar dos anos, em decorrência do avanço da medicina, da acentuada queda da taxa de fertilidade e mortalidade, sucedendo uma consequente elevação da expectativa de vida, além da também melhoria da qualidade de vida desta população (TEIXEIRA; OSELAME; NEVES, 2014; FERREIRA et al., 2017). Sabe-se que com o envelhecimento cronológico, há também o envelhecimento fisiológico do corpo humano, que reduz as funções dos órgãos, aumenta á vulnerabilidade e o risco do desenvolvimento de doenças crônicas, consequentemente aumentando as internações hospitalares. Este aumento se dá pelo envelhecimento populacional, aliado a melhor qualidade de vida e patologias inerentes ao processo histórico e da idade. O processo de envelhecimento tem por si só patologias inerentes, sabe-se que além do envelhecimento cronológico há também a associação das novas tecnologias (PITROWSKY; SHINOTSUKA; SOARES; LIMA; SALLUH, 2010; LUZ; BONIATTI, 2016; OLIVEIRA et al., 2017; FERREIRA et al., 2017).

Ao âmbito hospitalar as Unidades de Terapia Intensiva (UTI's) são denominadas como o setor onde pacientes hospitalizado em estado crítico e urgente, permanecem monitorados no decorrer da sua internação e progresso, O perfil predominante nestas unidades é de aproximadamente $60 \%$ de pacientes na terceira idade, caracterizado por pessoas com mais de 65 anos (CARBRERA; HARMÁNDEZ; CABRERA; PÉREZ; CASTELEIRO, 2015; SOUZA; BERSANETI; SIQUEIRA; MEIRA; BRUMATTI; PRADO, 2017; KREBS Et al., 2018; LUCENA et al., 2018; POLLIS et al., 2019).

Tratando-se de distúrbios psiquiátricos comumente acometidos em pacientes idosos hospitalizados destaca-se o delirium, uma síndrome que tem o nome derivado do latim latim "delirare", que significa, “estar fora do lugar”. Esta síndrome consiste em um estado de confusão aguda e retrata as manifestações da disfunção cerebral. Pode cursar com diferentes manifestações clinicas. Esta condição está associada a maior mortalidade em UTI's, devido ao ambiente ser um fator de risco para esta condição, pela ausência de iluminação natural, relógios e pelo isolamento do paciente (PAREJO, 2014; OLIVEIRA, et al. 2017; SOSA; ROBERTI; FRANCO; KLEINERT; PATRÓN; OSATNIK; BUEHLER, 2018). 
Em linhas gerais, a síndrome delirium apresenta-se de forma aguda, podendo o paciente, alterar o nível de consciência, apresentar déficit de atenção e percepção do ambiente, que demonstram serem as complicações frequentes com pacientes críticos, em consequência da exposição de episódios iatrogênicos (FARIA; MORENO, 2014; ITO; PEDRI, 2014; RODRIGUES; LAGE; BREMENKAMP; BOLLIS; MEIRELES; MORELATO, 2015).

Ainda há dificuldade no conhecimento desta síndrome, principalmente no que se refere aos processos fisiopatogênico e neuroquímico. No entanto sinais clínicos são evidenciados pela hiperatividade nos estados de agitação, pela hipoatividade no estado apático e pela interação de alterações difusas em episódios autoagressivos e para com os profissionais de saúde (SHINOTSUKA; SALLUH, 2014; BARROS; FIGUEIRÊDO; FERNANDES; NETO; COSTA, 2015; SILVA, 2016).

Para tal, a aplicação de instrumentos, escalas de avaliação e identificação, devem ser adotadas e implantadas nos serviços de saúde, com intuito de auxiliar em um possível diagnóstico, devendo ser realizado pela equipe multiprofissional, dentre eles, o enfermeiro. Desta forma, a equipe de enfermagem em conjunto com as demais equipes será fundamental na identificação dos fatores, e assim aplicar condutas baseadas em evidências, corroborando com a melhoria no quadro do paciente idoso (SHINOTSUKA; SALLUH, 2014; MOUZINHO; MADUREIRA, 2016; RODRIGUES; LAGE; BREMENKAMP; BOLLIS; MEIRELES; MORELATO, 2015).

Diante do exposto supracitado, considera-se importante o levantamento da magnitude desta problemática. Neste contexto, a presente revisão tem por objetivo ratificar os fatores de riscos e os cuidados de enfermagem na síndrome delirium nos pacientes idosos, assim como, buscar estratégias de prevenção no âmbito da Unidade de Terapia Intensiva.

\section{MATERIAIS E MÉTODOS}

Trata-se de um estudo descritivo exploratório com análise qualitativa através de uma revisão integrativa de literatura (VOSGERAU; ROMANOWSKI, 2014). A pesquisa ocorreu entre março e outubro de 2018, utilizou-se de artigos publicados e indexados nas bases de dados: Scientific Eletronic Library Online (Scielo) e o buscador Google acadêmico, Literatura Latino-Americana e do Caribe em Ciências de Saúde (LILACS) e Bireme.

Os critérios de inclusão empregados foram periódicos nacionais, originais, e revisões, entre os anos de 2013 e o primeiro semestre de 2018, que se encaixassem ao tema proposto. Utilizou-se para busca dos Descritores em Ciência da Saúde (DeCS): Pessoa Idoso, Delirium, Unidades de terapia intensiva, Enfermagem.

Foram excluídos manuscritos que não se adequaram à matriz metodológica proposta.

Para a elaboração da pesquisa realizada, através de leitura exploratória, foram selecionados os títulos e resumos, em seguida foi realizada uma análise desta leitura de forma ordenada e interpretativa 
com o intuito de fazer uma comparação entre os artigos. Foi realizado um fichamento dos dados obtidos para serem discutidos posteriormente com leituras atuais sobre a pesquisa.

Assim, o material composto foi de 21 artigos, os quais foram utilizados como amostra final de manuscritos para resultados e discussão e submetidos à técnica de avaliação e análise de conteúdo constituído por três etapas: exploração do referencial teórico, compilação e agrupamento de evidências e interpretação dos resultados.

A primeira etapa possibilitou uma visão geral do conteúdo dos artigos, por meio da leitura dos resumos e fichamento. Os textos na íntegra, após uma primeira leitura, foram organizados com o auxílio de um formulário composto das variáveis: ano/autor, objetivos, tipo de estudo e resultados encontrados.

A etapa de exploração do material foi desenvolvida a partir da releitura dos textos, culminando na construção de categorias temáticas de análise. Posteriormente, na etapa de interpretação dos resultados, foram observadas as colocações existentes sob a ótica de diferentes autores.

\section{RESULTADOS}

Os resultados obtidos demonstram intercessão entre os aspectos da síndrome do delírium da pessoa idosa hospitalizada na Unidade de Terapia Intensiva (UTI), como também nas causas e consequências dos fatores relacionados às estratégias de enfermagem diante da problemática na contemporaneidade conforme quadro 01.

Quadro 01 - Resultados encontrado com maior relevância.

\begin{tabular}{|c|c|c|c|c|}
\hline AUTOR & ANO & TÍTULO & OBJETIVO & RESULTADOS \\
\hline Faustino, et al. & 2016 & $\begin{array}{l}\text { Conhecimentos e } \\
\text { práticas da equipe } \\
\text { de enfermagem } \\
\text { para prevenção e } \\
\text { monitorização do } \\
\text { delirium em idosos. }\end{array}$ & $\begin{array}{l}\text { Verificar conhecimentos e } \\
\text { práticas da equipe de enferma- } \\
\text { gem de uma unidade de terapia } \\
\text { intensiva (UTI) acerca do cui- } \\
\text { dado ao idoso para prevenção e } \\
\text { monitorização do delirium. }\end{array}$ & $\begin{array}{l}\text { Dados demonstraram conhecimento } \\
\text { superficial sobre a manifestação do } \\
\text { delirium, expresso por agitação, falas } \\
\text { desconexas, alucinações e perda súbita } \\
\text { de memória. }\end{array}$ \\
\hline Luz e Boniatti. & 2016 & $\begin{array}{l}\text { Associação do } \\
\text { delirium com } \\
\text { cognição, capaci- } \\
\text { dade funcional e } \\
\text { qualidade de vida } \\
\text { no paciente crítico. }\end{array}$ & $\begin{array}{l}\text { Investigar se há associação com } \\
\text { qualidade de vida, capacidade } \\
\text { funcional e cognição do idoso. }\end{array}$ & $\begin{array}{l}\text { Foram incluídos no estudo } 88 \text { pacientes. } \\
\text { A incidência de delirium foi } 15,9 \% \text { com } \\
\text { um tempo médio de duração de } 3,7 \pm 5,1 \\
\text { dias. Com relação à cognição na alta hos- } \\
\text { pitalar, houve uma tendência de redução } \\
\text { no escore do mini-exame mental para os } \\
\text { pacientes com delirium em comparação } \\
\text { aos pacientes sem delirium. }\end{array}$ \\
\hline
\end{tabular}




\begin{tabular}{|c|c|c|c|c|}
\hline Mori, et al. & 2016 & $\begin{array}{l}\text { Incidência e fatores } \\
\text { relacionados ao de- } \\
\text { lirium em unidade } \\
\text { de terapia intensiva. }\end{array}$ & $\begin{array}{l}\text { Identificar a incidência de deli- } \\
\text { rium. Comparar as característi- } \\
\text { cas demográficas e clínicas dos } \\
\text { pacientes com e sem delirium } \\
\text { e verificar os fatores relaciona- } \\
\text { dos ao delirium em pacientes } \\
\text { internados em unidade de } \\
\text { terapia intensiva (UTI). }\end{array}$ & $\begin{array}{l}\text { Do total de } 149 \text { pacientes da amostra, } 69 \\
(46,3 \%) \text { apresentaram delirium durante a } \\
\text { internação na UTI, observando-se que a } \\
\text { média da idade, o índice de gravidade e } \\
\text { o tempo de permanência nas UTI foram } \\
\text { estatisticamente maiores. Os fatores } \\
\text { relacionados ao delirium foram: idade, } \\
\text { midazolam, morfina e propofol. }\end{array}$ \\
\hline Barros, et al. & 2015 & $\begin{array}{l}\text { Delirium em idosos } \\
\text { em unidades de } \\
\text { terapia intensiva: } \\
\text { revisão integrativa } \\
\text { da literatura. }\end{array}$ & $\begin{array}{l}\text { Realizar um levantamento } \\
\text { acerca do delirium em idosos } \\
\text { em unidade de terapia intensiva } \\
\text { (UTI). }\end{array}$ & $\begin{array}{l}\text { Foram encontradas } 68 \text { produções e sele- } \\
\text { cionadas } \\
\text { 16, as quais foram incidência e prevalência } \\
\text { de delirium em idosos, fatores de risco } \\
\text { precipitantes e predisponentes do delirium } \\
\text { em idoso, estratégias de prevenção de } \\
\text { fatores de risco modificáveis do delirium. }\end{array}$ \\
\hline Ribeiro, et al. & 2015 & $\begin{array}{l}\text { Conhecimentos } \\
\text { dos enfermeiros } \\
\text { sobre delirium no } \\
\text { paciente criticam: } \\
\text { discurso do sujeito } \\
\text { coletivo. }\end{array}$ & $\begin{array}{l}\text { Analisar o conhecimento de } \\
\text { enfermeiros de uma unidade } \\
\text { de terapia intensiva acerca do } \\
\text { delirium no paciente crítico. }\end{array}$ & $\begin{array}{l}\text { Da análise dos dados emergiram sinais } \\
\text { e sintomas; uso de sedativos; contenção } \\
\text { física; ambiente; falta de preparo profissio- } \\
\text { nal. Verificou-se que os enfermeiros têm } \\
\text { dúvidas sobre o delirium, seu manejo em } \\
\text { terapia intensiva, utilização de escalas e in- } \\
\text { tervenções de enfermagem. Aponta-se para } \\
\text { a necessidade de educação sobre o tema. }\end{array}$ \\
\hline Silva. & 2016 & $\begin{array}{l}\text { Dificuldade diag- } \\
\text { nóstica dos profis- } \\
\text { sionais da saúde } \\
\text { frente ao delirium: } \\
\text { uma revisão de } \\
\text { literatura. }\end{array}$ & $\begin{array}{l}\text { Identificar na literatura as } \\
\text { dificuldades em diagnosti- } \\
\text { car precocemente o paciente } \\
\text { com delirium, e quais são } \\
\text { as condutas realizadas pelos } \\
\text { profissionais de saúde quando o } \\
\text { diagnóstico é feito. }\end{array}$ & $\begin{array}{l}\text { Os resultados encontrados denotam que, } \\
\text { por vezes, este distúrbio não é reconhe- } \\
\text { cido adequadamente e em tempo hábil de } \\
\text { tratamento. Outro fator relevante encon- } \\
\text { trado é a prevalência de vários riscos em } \\
\text { idosos hospitalizados. Consideramos que } \\
\text { pacientes em delirium devem ser cons- } \\
\text { tantemente avaliados, mesmo que não } \\
\text { apresentem queixas, pois este distúrbio } \\
\text { é oscilante e pode não ser diagnosticado } \\
\text { nos primeiros atendimentos. }\end{array}$ \\
\hline $\begin{array}{l}\text { Rodrigues, } \\
\text { et al. }\end{array}$ & 2015 & $\begin{array}{l}\text { Frequência de } \\
\text { medicamentos } \\
\text { potencialmente } \\
\text { inapropriados em } \\
\text { pacientes interna- } \\
\text { dos com delirium. }\end{array}$ & $\begin{array}{l}\text { Identificar medicamentos } \\
\text { potencialmente causadores de } \\
\text { delirium em pacientes idosos } \\
\text { portadores desta síndrome e } \\
\text { seu risco para mortalidade } \\
\text { durante a hospitalização. }\end{array}$ & $\begin{array}{l}\text { Aproximadamente } 30 \text { pacientes }(58,82 \%) \\
\text { faziam uso de fármacos de risco para deli- } \\
\text { rium, sendo que } 39,2 \% \text { utilizavam medica- } \\
\text { mentos considerados de alto risco e } 13,7 \% \\
\text { usavam concomitantemente três fármacos. } \\
\text { Dentre as medicações de alto risco, os } \\
\text { benzodiazepínicos foram empregados em } \\
23,5 \% \text { dos pacientes. O tempo médio de } \\
\text { internação foi de } 24 \pm 18 \text { (1-86) dias e } 25 \\
(49 \%) \text { pacientes evoluíram para o óbito. }\end{array}$ \\
\hline
\end{tabular}

Fonte: Autores, 2018. 


\section{DISCUSSÃO}

\section{Fatores de risco e estratégia de prevenção: enfermeiro e equipe multiprofissional}

Conforme quadro 01, vale salientar através da presente revisão, corroborando com destaque dos autores, que dados acerca do delirium, em especial na pessoa idosa, apresentam-se escassos. Desta forma, além de abordar uma temática pouca investigada, ressalta-se que a síndrome clínica está atrelada a vários fatores de risco (SOUZA; BERSANETI; SIQUEIRA; MEIRA; BRUMATTI; PRADO, 2017; CHAGAS; BORGES; CHAGAS, 2016).

Contudo, a pessoa idosa apresenta dificuldades na sua identificação acerca desta síndrome, pela equipe multiprofissional, neste contexto a desorientação e confusão mental, comumente observado aos idosos internados, advêm a sinais sugestivos do delirium, no entanto tendem a passar despercebidos. Portanto, observam-se em cuidados críticos, falhas no diagnóstico do delirium, mas em contrapartida há aumento nas taxas de permanência hospitalar e morbimortalidade em delirium (LUZ; BONIATTI, 2016; MORI; TAKEDA; CARRARA; COHRS; ZANEI; WHITAKER, 2016).

Em análise, a temática se destacou com uma gama de fatores de risco relacionados ao delirium, os quais se apresentam como não modificáveis, ponderando as condições já existentes da pessoa idosa internada, destas salientou-se, a idade avançada, o cognitivo, a abstinência ao tabagismo e consumo de álcool (ZANINI, 2010; FARIA; MORENO, 2014; ITO; PEDRI, 2014; CHAGAS; BORGES; CHAGAS, 2016).

Contrapondo, há a existência de fatores de risco modificáveis, quais se apresentam a condições clinicas agudas, podendo ocorrer intervenções quando evidenciaram-se aspectos como avaliação prévia na admissão de urgência, dor, contenção física, sedativos e analgésicos opióides administrados, infecções, hipóxia, distúrbios do sono, ambiente da UTI como iluminação artificial, ruídos, isolamento familiar, entre outras variáveis existentes no processo de hospitalização (LUZ; BONIATTI, 2016; MORI; TAKEDA; CARRARA; COHRS; ZANEI; WHITAKER, 2016).

A síndrome delirium tem relação direta com o agravamento de comorbidades crônicas, as quais podem apresentar sinais de disfunção cardiovascular, hepática e renal. Contudo, o reconhecimento precoce dos fatores de risco modificáveis mostra-se como a estratégia fundamental de prevenção do delirium em idosos em âmbito de UTI's (GUSMAO-FLORES, 2014; RIBEIRO; NASCIMENTO; LAZZARI; JUNG; BOES; BERTONCELLO, 2015).

Os autores retratam em suas pesquisas que fatores de risco modificáveis transitam entre os aspectos farmacológico e não farmacológico, são importantes na melhora ao manejo do quadro clinico pois, farmacologicamente a utilização de haloperidol, e o sedativo dexmetomidina, apresentam redução da problemática eminente (SHINOTSUKA; SALLUH, 2014; BARROS; FIGUEIRÊDO; FERNANDES; NETO; COSTA, 2015; SILVA, 2016). 
Para tal, as estratégias não farmacológicas, desenham-se como estimulação cognitiva, mobilização precoce, o profissional psicólogo na UTI, o familiar do idoso, a promoção de ambiente calmo, silencioso e com redução de estímulos sonoros, entre outros aspectos possíveis quais já foram evidenciados (FARIA; MORENO, 2014; ITO; PEDRI, 2014; RODRIGUES; LAGE; BREMENKAMP; BOLLIS; MEIRELES; MORELATO, 2015).

Estudos apontam a incidência de delirium a pacientes com faixa etária acima dos 60 anos, fenômeno que rapidamente acomete os idosos, fazendo com que precisem de cuidados em ambientes críticos, portanto, constatando-se que a ocorrência da síndrome apresenta impacto significativo na mortalidade (FARIA; MORENO, 2014; ITO; PEDRI, 2014; RODRIGUES; LAGE; BREMENKAMP; BOLLIS; MEIRELES; MORELATO, 2015).

Vimos então que a temática é relevante na sobrevida e na alteração cognitiva de longo prazo deste idoso hospitalizado e assim reconhecer os fatores associados ao aparecimento é fundamental a toda equipe multiprofissional, identificando os idosos propensos a desenvolver o delirium, déficit cognitivo prévio e demências, fazendo com que a equipe de enfermagem seja o instrumento preponderante na observação de gatilhos e intervenções (PAREJO, 2014; SOSA; ROBERTI; FRANCO; KLEINERT; PATRÓN; OSATNIK; BUEHLER, 2018).

Desta forma, quando a demência é associada ao delirium, há que se aplicar o melhor manejo na prática assistencial de enfermagem, pois os pacientes apresentam disfunções e piora significativa da cognição. Por isso, a atuação da equipe de enfermagem deve apresentar ações preventivas, culminando em estratégias de intervenção precoce (CARBRERA; HARMÁNDEZ; CABRERA; PÉREZ; CASTELEIRO, 2015; SOUZA; BERSANETI; SIQUEIRA; MEIRA; BRUMATTI; PRADO, 2017; KREBS et al., 2018).

Contudo, ao que tange a prevenção farmacológica, a utilização do haloperidol, a redução de benzodiazepínicos e as alternativas à terapia farmacológica sedativa são evidenciadas, porem recomenda-se o uso de haloperidol nos idosos, em apenas metade da dose utilizada em adultos e se persistir após o uso, é observado o risco de esta medicação converter em um estado hiperativo da síndrome para um estado hipoativo (PITROWSKY; SHINOTSUKA; SOARES; LIMA; SALLUH, 2010; LUZ; BONIATTI, 2016; MORI; TAKEDA; CARRARA; COHRS; ZANEI; WHITAKER, 2016).

Entretanto, a utilização de medicamentos psicoativos pode interferir na neurotransmissão cerebral e induzir os sintomas do delirium. Os autores apontam que no delirium, os benzodiazepínicos e opióides são comuns nos protocolos de sedação em UTI's. Como alternativa de droga para a sedação em risco de delirium, a dexmetomidina, contribuiu para diminuição na incidência, prevalência e mortalidade de delirium, segundo outros autores (PITROWSKY; SHINOTSUKA; SOARES; LIMA; SALLUH, 2010; OLIVEIRA, et al. 2017; PAREJO, 2014; KREBS et al., 2018).

Tratando-se de estratégias não farmacológicas de prevenção do delirium, a contenção física, tem por objetivo a não retirada de cateteres venosos, tubos e precaução de lesões autoinfrigidas, privação do sono e pacientes que sofrem com o isolamento familiar, são evidenciados. Em nível 
nacional, por meio do estatuto do idoso, ele tem direito a presença de familiares e/ou acompanhantes em seu período de internação, é garantido dando ênfase à humanização (GUSMAO-FLORES, 2014; CARBRERA; HARMÁNDEZ; CABRERA; PÉREZ; CASTELEIRO, 2015; RIBEIRO; NASCIMENTO; LAZZARI; JUNG; BOES; BERTONCELLO, 2015).

A existência de uma padronização de medidas protocolos e/ou diretrizes, é necessária para eficiência da prática assistencial da enfermagem aliada a uniformização dos cuidados, otimizando os resultados, tornando prioritário a melhora do quadro do paciente. Para tal, na identificação do diagnóstico do delirium, há instrumentação do CAM-ICU, e outros facilitadores na identificação da problemática (BARROS; FIGUEIRÊDO; FERNANDES; NETO; COSTA, 2015; FAUSTINO; PEDREIRA; SILVA; FREITAS, 2016; SILVA, 2016).

O profissional enfermeiro e equipe vigilante a beira leito, devem identificar as alterações comportamentais do idoso e a evolução do seu quadro, no entanto, é visto que, a falta de conhecimento do profissional, a falta de tempo e de interesse, dentre outras razões, são evidenciadas. Deste modo a equipe de enfermagem tem como primazia estratégias de educação permanente nesta temática, para obter excelência no processo de cuidar (ZANINI, 2010; MOUZINHO; MADUREIRA, 2016; FAUSTINO; PEDREIRA; SILVA; FREITAS, 2016; SOSA; ROBERTI; FRANCO; KLEINERT; PATRÓN; OSATNIK; BUEHLER, 2018).

\section{CONCLUSÃO}

A reflexão e compreensão dos aspectos de interfaces sobre os fatores de riscos e cuidados de enfermagem na síndrome delirium em idosos, assim como em estratégias de prevenção no âmbito da UTI e suas dimensões na assistência ao paciente, estimulando e evidenciando a importância do profissional enfermeiro, foram alcançados, e seus resultados, através desta investigação apresentam relevância social, profissional e acadêmica.

Ao que tange a síndrome, quase que unanimemente, os autores apresentam a temática como algo a ser mais evidenciado e esclarecido estratificando as lacunas ao trazer respostas a luz deste tema, propor avanços no reconhecimento da síndrome na prática assistencial e refletir sobre seus resultados em pesquisas.

Traz ainda, a observância do grande número de incidência de delirium a pessoa idosa, associados aos fatores passíveis de mudanças, pelo meio de estratégias de prevenção. Ressalta-se ainda a necessidade de novas práticas observacionais e assistenciais não só pela equipe de enfermagem, mas por toda equipe multiprofissional.

Neste contexto, conclui-se que é de fundamental importância a identificação dos sinais nos idosos e sua rápida intervenção e diagnóstico, para que assim possa ser proposta uma conduta adequada aos episódios, estabelecendo ações de vigilância continua. 
Portanto, com a reflexão desta temática, atrelada às atribuições da enfermagem, objetivamos contribuir ao compreendimento da incidência e prevalência da síndrome, proporcionando clareamento sobre a importância dos fatores de risco e estratégias de prevenção modificáveis do delirium em UTI, e como consequência a melhora no quadro clinico, e a eficiência na assistência em enfermagem tão necessária.

Mesmo com relatos do crescimento do alento à problemática, percebeu-se que há escassez da literatura sobre a luz da temática, o que resultou na principal limitação deste estudo. Desta forma, evidencia-se a necessidade da realização de novos estudos a fim de investigar e analisar, os processos da síndrome do delirium e o manejo da enfermagem e suas dimensões na assistência ao paciente.

\section{AGRADECIMENTOS}

Agradecimento a excelentíssima enfermeira, professora, mestre em engenharia biomédica, Janayna do Rocio Luvizotto, coordenadora do curso de enfermagem do Centro Universitário Campos de Andrade - UNIANDRADE, pela orientação do projeto inicial e instruções sobre a temática.

\section{REFERÊNCIAS}

BARROS, Marcia Abath Aires de, et al. Delirium em idosos em unidades de terapia intensiva: revisão integrativa da literatura. Revista de Pesquisa Cuidado é Fundamental Online, 2015, 7.3: 2738-2748.

CARBRERA, Ángel Julio Romero, et al. Guía de práctica clínica para la atención al adulto mayor hospitalizado con delirium. MediSur, 2015, 13.3: 458-466.

CHAGAS, Natália Mota S.; BORGES, Daniel G. Suzuki; CHAGAS, Marcos Hortes N. Delirium como fator de risco para demência em idosos: uma atualização. J Bras Psiquiatria, 2016, 65.1: 94-8.

FARIA, R. S.; MORENO, Rui Paulo. Delirium na unidade de cuidados intensivos: uma realidade subdiagnosticada. Rev Bras Ter Intensiva, 2013, 25.2: 137-47.

FAUSTINO, Tássia Nery, et al. Conhecimentos e práticas da equipe de enfermagem para prevenção e monitorização do delirium em idosos. Revista Baiana de Enfermagem, 2016, 30.3.

FERREIRA, Wellington Fernando da Silva, et al. Urgência e emergência nas dimensões da pessoa idosa: Sistema Manchester. Revista Saúde e Desenvolvimento, 2017, 11.6: 283-293. 
FERREIRA, Wellington Fernando da Silva, et al. Direitos humanos da pessoa idosa portadora de esquizofrenia: Uma contribuição da enfermagem. Revista Saúde e Desenvolvimento, 2017, 11.6: 219-229.

GUSMAO-FLORES, Dimitri. Escalas de avaliação de delirium em pacientes graves: revisão sistemática da literatura. Rev Bras Ter Intensiva, 2013, 25.2: 148-154.

ITO, Christian Makoto; PEDRI, Lucas Eduardo. O uso da terapia farmacológica para a profilaxia do delirium: revisão sistemática. "Programa Mais Médicos” e a Saúde no Brasil, 2013, 11.4: 391-5.

KREBS, Jessica Aline, et al. A influência do Delirium no tempo de ventilação mecânica em pacientes críticos: uma revisão sistemática. ABCS Health Sciences, 2018, 43.1.

LUCENA, Cristiane Gislaine, et al. Percepção dos acadêmicos de enfermagem sobre o transtorno afetivo bipolar. Ciência \& Desenvolvimento-Revista Eletrônica da FAINOR, 2018, 11.2.

LUZ, Lúcia Fabiane Da Silva; BONIATTI, Márcio Manozzo. Associação do delirium com cognição, capacidade funcional e qualidade de vida no paciente crítico. SEFIC 2016, 2017.

MORI, Satomi, et al. Incidência e fatores relacionados ao delirium em Unidade de Terapia Intensiva. Revista da Escola de Enfermagem da USP, 2016, 50.4: 587-593.

MOUZINHO, Isabela Taveira; FARIA, Marcos Quirino Gomes; madureira, eduardo miguel prata. Ocorrência de delirium como complicação hospitalar em idosos no pós-operatório de fratura de fêmur. Revista Thêma et Scientia, 2016, 6.2: 237-247.

OLIVEIRA, Edilaine Cristina de, et al. Cuidados pós-alta em pacientes idosos com sequelas de acidente vascular cerebral: planejamento de alta hospitalar. Revista Saúde e Desenvolvimento, 2017, 11.9: $172-197$.

OLIVEIRA, Samara de, et al. O enfrentamento da equipe de enfermagem em atendimentos a pacientes em crise psicótica. Revista de Atenção à Saúde (antiga Rev. Bras. Ciên. Saúde), 2017, 15.53: 50-56.

PAREJO, Lucinéia Stach. Delirium como foco da atenção para os enfermeiros de terapia intensiva [mestrado]. Botucatu (SP): Universidade Estadual Paulista Júlio de Mesquita Filho, 2014. 
PITROWSKY, Melissa Tassano, et al. Importância da monitorização do delirium na unidade de terapia intensiva. Revista Brasileira de Terapia Intensiva, 2010, 22.3: 274-279.

POLLIS, Ariane Alves, et al. Transtorno de personalidade borderline e assistência de enfermagem na emergência psiquiátrica. Disciplinarum Scientia Saúde, 2019, 20.1: 15-36.

RIBEIRO, Simone Cidade Lima, et al. Conhecimento de Enfermeiros sobre Delirium no paciente crítico: discurso do sujeito coletivo. Enferm. Florianópolis, 2015, 24.2: 513-20.

RODRIGUES, Luara Ramos, et al. Frequência de medicamentos potencialmente inapropriados em pacientes internados com delirium. Rev. Soc. Bras. Clín. Méd, 2015, 13.1.

SHINOTSUKA, Cassia Righy; SALLUH, J. I. Percepções e práticas sobre delirium, sedação e analgesia em pacientes críticos: uma revisão narrativa. Rev Bras Ter Intensiva, 2013, 25.2: 155-61.

SILVA, CL. Dificuldade diagnóstica dos profissionais da saúde frente ao delirium: uma revisão de literatura. Saúde. Com, 2016;9(4).

SOSA, Fernando Ariel, et al. Avaliação de delirium com uso do modelo PRE-DELIRIC em uma unidade de terapia intensiva na Argentina. Rev Bras Ter Intensiva, 2018, 30.1: 50-56.

SOUZA, Regina Claudia, et al. Capacitação de enfermeiros na utilização de um instrumento de avaliação de delirium. Revista Gaúcha de Enfermagem, 2017, 38.1.

TEIXEIRA, Valdeci De Assis; OSELAME, Gleidson Brandão; NEVES, Eduardo Borba. O Protocolo de Manchester no sistema único de saúde e a atuação do enfermeiro. Revista da Universidade Vale do Rio Verde, 2014, 12.2: 905-920.

VOSGERAU, Dilmeire Sant'Anna Ramos; ROMANOWSKI, Joana Paulin. Estudos de revisão: implicações conceituais e metodológicas. Revista diálogo educacional, 2014, 14.41: 165-189.

ZANINI, Rachel Schlindwein. Demência no idoso. Revista Neurociências, 2010, 18.2: 220-226. 
